\title{
Review Article \\ Towards Understanding Extracellular ROS Sensory and Signaling Systems in Plants
}

\author{
Saijaliisa Kangasjärvi ${ }^{1}$ and Jaakko Kangasjärvi ${ }^{2,3}$ \\ ${ }^{1}$ Department of Biochemistry, University of Turku, 20014 Turku, Finland \\ ${ }^{2}$ Department of Biosciences, University of Helsinki, P.O. Box 65, 00014 Helsinki, Finland \\ ${ }^{3}$ Distinguished Scientist Fellowship Program, College of Science, King Saud University, P.O. Box 2455, Riyadh 11451, Saudi Arabia
}

Correspondence should be addressed to Jaakko Kangasjärvi; jaakko.kangasjarvi@helsinki.fi

Received 23 February 2014; Revised 11 May 2014; Accepted 14 May 2014; Published 1 June 2014

Academic Editor: Scott Finlayson

Copyright ( 2014 S. Kangasjärvi and J. Kangasjärvi. This is an open access article distributed under the Creative Commons Attribution License, which permits unrestricted use, distribution, and reproduction in any medium, provided the original work is properly cited.

\begin{abstract}
Reactive Oxygen Species (ROS) are ubiquitous metabolites in all aerobic organisms. Traditionally ROS have been considered as harmful, accidental byproducts of cellular functions involving electron transport chains or electron transfer. However, it is now recognized that controlled production of ROS has significant signaling functions, for example, in pathogen defense, in the regulation of stomatal closure, or in cell-to-cell signaling. ROS formation in subcellular compartments is critical to act as "alarm" signal in the response to stress, and the concept of ROS as primarily signaling substances has emerged. The involvement of ROS in several developmental and inducible processes implies that there must be coordinated function of signaling network(s) that govern ROS responses and subsequent processes. The air pollutant ozone can be used as a useful tool to elucidate the function of apoplastic ROS: $\mathrm{O}_{3}$ degrades in cell wall into various ROS which are interpreted as ROS with signaling function inducing downstream responses. We have used ozone as a tool in mutant screens and transcript profiling-reverse genetics to identify genes involved in processes related to the signaling function of ROS. We review here our recent findings in the elucidation of apoplastic ROS sensing, signaling, and interaction with various symplastic components.
\end{abstract}

\section{Introduction}

Reactive Oxygen Species (ROS) are ubiquitous metabolites in all aerobic organisms. ROS include superoxide $\left(\cdot \mathrm{O}_{2}{ }^{-}\right)$, hydrogen peroxide $\left(\mathrm{H}_{2} \mathrm{O}_{2}\right)$, hydroxyl radical $(\bullet \mathrm{OH})$, singlet oxygen $\left({ }^{1} \mathrm{O}_{2}\right)$, and the air pollutant ozone $\left(\mathrm{O}_{3}\right)$. Traditionally ROS have been considered as harmful, accidental byproducts of cellular functions involving electron transport chains or electron transfer, where electrons are accidentally passed to molecular oxygen. Consequently, cells have effective enzymatic and chemical antioxidative defenses to remove these ROS $[1,2]$. The most prominent sources of ROS in plant cells are thought to be chloroplasts and mitochondria, which both contain electron transfer chains involving multiple components. Processes that take place in peroxisomes and cytoplasm also produce ROS and the apoplast can be an important site for ROS generation, although the quantity of ROS produced is several-fold less than that of the organelles.
However, it is now recognized that controlled production of ROS, for example, by the plasma membrane-localized NADPH oxidases, which produce in a controlled fashion - $\mathrm{O}_{2}{ }^{-}$to the apoplastic space, has significant signaling functions [3-5]. ROS formation in other subcellular compartments, for example, chloroplasts, mitochondria, and peroxisomes is critical to act as "alarm" signal in the response to stress, and the concept of ROS as primarily signaling substances has emerged [2,6-10]. The involvement of ROS in several developmental and inducible processes implies that there must be coordinated function of signaling network(s) that govern ROS responses and subsequent processes, although detailed descriptions of how such interactions work are lacking [11]. Furthermore, the perception of ROS produced for signaling functions is still almost completely unknown in any species.

During the last two decades different artificial systems have been developed to induce ROS production in different subcellular compartments by noninvasive methods. 
These include transgenic plants where the activity of the nonenzymatic or enzymatic antioxidative systems has been decreased, or where the peroxisomal catalase activity has been prevented by antisense methodology, or by expressing the $\mathrm{H}_{2} \mathrm{O}_{2}$-producing enzyme glycolate oxidase in the chloroplast, thus increasing the peroxisomal or chloroplastic ROS production under photorespiratory conditions $[7,12-$ 15]. These approaches have significantly increased the understanding of the mechanisms where intracellular ROS act as signaling substances. However, these approaches cannot be used to elucidate the role, significance, or the mechanisms involved in apoplastic ROS signaling and the significance of, for example, the ROS production by the plasma membrane NADPH oxidases. We have addressed this during the last two decades in noninvasive ways by using the gaseous threeatomic form of oxygen, ozone, which is itself also a ROS $[6,16,17]$.

Ozone $\left(\mathrm{O}_{3}\right)$ is an air pollutant produced in the atmosphere as a result of human activities. In high concentrations it causes visible damage in the affected leaves [18, 19]. The lesions visible in ozone-exposed leaves are a result of programmed cell death induced by the ROS produced from ozone degradation in the apoplastic space in the affected leaf cells $[6,16,20,21]$. Because of this, ozone can also be used as a useful tool to probe and elucidate the function of apoplastic ROS production with the following rationale: $\mathrm{O}_{3}$ degrades into various ROS in the extracellular space of plant leaf cells; ROS formed from $\mathrm{O}_{3}$ breakdown are interpreted by the plant cells as apoplastic ROS with signaling function, which induces downstream responses $[16,22]$. This makes $\mathrm{O}_{3}$ a convenient tool to study the action of apoplastic ROS in a noninvasive way (Figure 1). We have used ozone as a tool in mutant screens and transcript profiling-reverse genetics in A. thaliana and identified genes encoding proteins involved in processes related to the signaling function of ROS, such as the transcription factor-interacting nuclear protein $\mathrm{RCD1}$, the chloroplast envelope membrane protein RETICULATA (RE; originally identified as RCD2), a central stomatal regulator, the long sought-after guard cell anion channel SLAC1 (originally $r c d 3$ ), and an extracellular protein GRI (originally $r c d 5$ ) possibly involved in ROS sensing [2326]. The same approach has also identified key components in the ascorbic acid (vitamin C) biosynthesis [27, 28].

Our mutant screens for ozone sensitivity have revealed new components in cell signaling. For example, the guard cell anion channel was not found in any direct approaches for guard cell processes-it was found in three independent mutant screens of which two were for increased ozone sensitivity and one for $\mathrm{CO}_{2}$ insensitivity [24, 29, 30]. Furthermore, we showed in a large ecotype screen good correlation between visible ozone damage and initial stomatal conductance/ozone dose that enters leaves during the very first moments of exposure [31]. Thus, ozone sensitivity is also a good marker for altered stomatal behavior and therefore can be used as a proxy for identifying components in early stomatal signaling downstream of apoplastic ROS produced normally as a result of NADPH oxidase activation. Some components upstream of the central regulatory component of stomatal movements have been identified. These include the anion

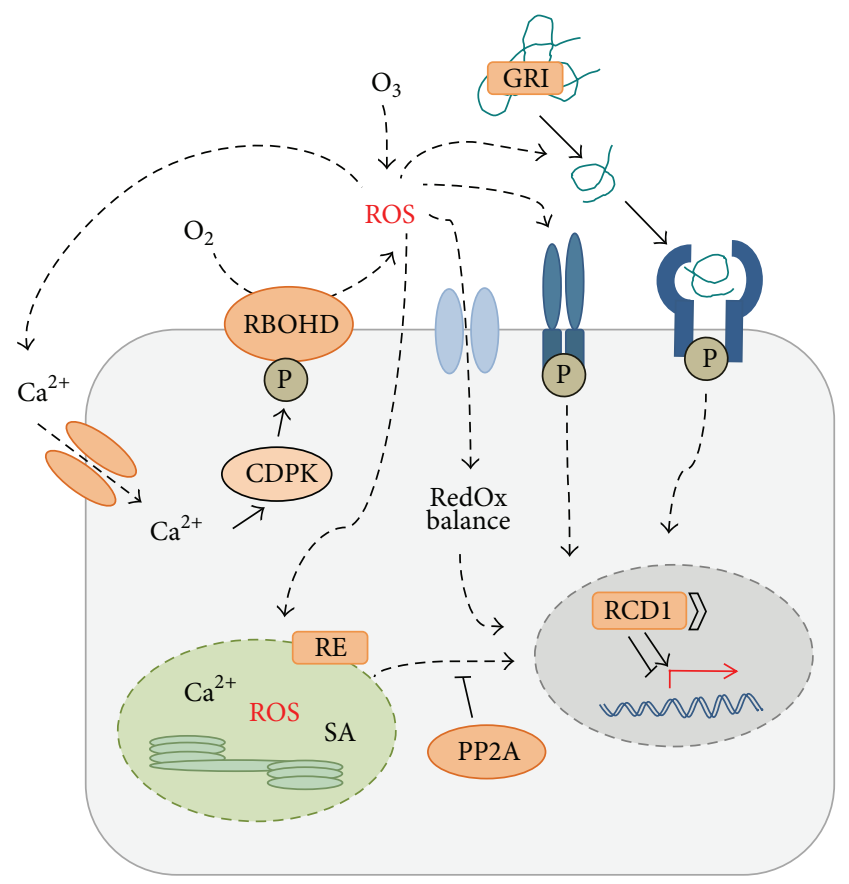

FIGURE 1: Apoplastic ROS sensory systems and downstream signaling. The action and role of ROS in the apoplast can be addressed by exposing plants to ozone $\left(\mathrm{O}_{3}\right)$ which degrades in the cell wall to the same ROS that are produced by the activity of the plasma membrane NADPH oxidases (exemplified here by the D-isoform RBOHD). The ROS can be sensed by direct modification of plasma membrane proteins, for example, Receptor-like kinases (RLK) or ion channels, indirectly by RLKs by ROS-dependent modification of cell wall components, exemplified here by modification off a cell wall protein GRI $[26,33]$, or by transport of $\mathrm{H}_{2} \mathrm{O}_{2}$ through aquaporins. The apoplastic ROS signal can be transmitted directly to nucleus and/or amplified in the chloroplast, followed by retrograde signaling where the chloroplast envelope protein RETICULATA (RE) and the cytoplasmic type 2A protein phosphatases (PP2A) appear to be involved. In the nucleus specific transcription factors and coregulators (exemplified here by RCD1) specifically activate or inactivate target genes and create the apoplastic ROS-dependent transcriptional pattern [8].

channel SLAC1, the protein kinase OST1 and the protein phosphatases $A B I 1$ and $A B I 2$ interacting with it, and the ABA coreceptor proteins PYR/PYL/RCAR. However, several components, including the mechanisms for ROS sensing and $\mathrm{Ca}^{2+}$-related sensory and signaling components-including the plasma membrane $\mathrm{Ca}^{2+}$ influx channel-which is activated by apoplastic ROS/ozone exposure, have remained unidentified [32] and are thus a challenging target for future studies.

\section{Apoplastic ROS Sensory Systems in Plants}

During the last several years there has been general interest in the elucidation of the role of ROS as signaling substances and in the identification of the extracellular ROS sensing/perception systems and the immediate downstream signaling. It has been shown recently [36] that ROS act in 
local cell-to-cell signaling in relay manner. This has been named as "the ROS wave" that spreads relatively fast, about $8 \mathrm{~cm} /$ minute. The concept of "ROS wave" requires perception or sensing of ROS produced in the neighboring cells. However, ROS perception and sensing in the extracellular compartment are almost unknown in any organism. At least five different mechanisms can be proposed to be involved in apoplastic ROS sensing. Four of these are presented in Figure 1: (1) perception by an apoplastic receptor protein, which is directly modified by the ROS, or which senses ROS-induced modifications in another component present in the apoplast. We have shown indirect evidence that small apoplastic proteins [36] and plasma membrane RLKs [37, 38] can be involved in apoplastic ROS sensing. (2) Some plasma membrane ion channels, such as the $\mathrm{K}^{+}$-channel SKOR [39], are direct targets of redox regulation. It has been shown that specific $\mathrm{Ca}^{2+}$-dependent protein kinases are involved in ROS signaling. Apoplastic ROS, including $\mathrm{O}_{3}$ exposure, induce an immediate activation of a still unidentified plasma membrane $\mathrm{Ca}^{2+}$ channel [40,41], which could as well be a target for regulation by ROS in a manner similar to SKOR. Cys residues in the channel protein itself, or other regulatory components involved in the activation of the channel extra- or intracellularly would affect $\mathrm{Ca}^{2+}$ fluxes and thus, in essence, form a sensory system for apoplastic ROS linking them directly to the activation of the downstream $\mathrm{Ca}^{2+}$-dependent protein kinases. (3) Direct transport of apoplast-formed $\mathrm{H}_{2} \mathrm{O}_{2}$ via aquaporins, followed by sensing in the symplasm, as has been shown for the mammalian NADPH oxidase-produced ROS. (4) There is a change in the total cellular redox balance as a result of action of the ROS on redox-active substances. The fifth possibility is the oxidation of plasma membrane lipids, which results in the formation of lipid-based signaling molecules that are further sensed. These five alternatives cannot be regarded as exclusive since they may all be involved in the various individual responses elicited by $\mathrm{O}_{3}$ in the cells affected. The identification and further elucidation of the sensory mechanisms for ROS present one of the most significant future challenges in ROS biology.

To specifically study and elucidate the mechanisms and components involved in apoplastic ROS sensing and the immediate downstream responses, a fast and specific response that can be easily observed is needed. We have shown that a rapid transient decrease in stomatal conductance (RTD) is triggered by ROS formed from the breakdown of $\mathrm{O}_{3}$ in the cell wall of guard cells. RTD is ABA independent but requires the activity of the protein kinase SnRK2.6/OST1, involving the $\mathrm{PP} 2 \mathrm{C}$ type protein phosphatases $\mathrm{ABI}$ and ABI2, and the anion channel SLAC1 [42]. The formation of ROS from $\mathrm{O}_{3}$ in the guard cell apoplast mimics the ROS producing activity of the plasma membrane NADPH oxidases AtrbohD and AtrbohF. These NADPH oxidases are also activated in the guard cells, for example, by perception of the bacterial flagellin (or the flg22 peptide in experimental systems) by the flagellin receptor FLS2, which leads to rapid stomatal closure [43]. It can be hypothesized that the apoplastic ROS formation can act as an overriding mechanism that rapidly closes the stomatal pore, which is visible as the RTD induced by ROS formation from a short pulse of ozone. This fast response should be a useful marker in mutant screens and other approaches to identify components involved in ROS sensing and immediate downstream signaling.

Receptor-like kinases (RLKs) and transmembrane proteins that perceive signals with their extracellular domains and propagate them through the intracellular kinase domains are important components of many signaling networks. In plants RLKs control developmental and hormone responses, stomatal regulation, and stress responses, as well as defense against bacterial and fungal pathogens [44-46]. Arabidopsis has more than 600 members in the RLK gene family. Although RLKs have been implicated in numerous contexts, defined functions have been assigned to only a few members in plants. ROS production and redox changes in the apoplast might also be perceived by extracellular proteins and/or plasma membrane-localized receptors (Figure 1). However, the identity and function of these receptors have so far remained elusive. One of the RLK groups, CYSTEINE-RICH RECEPTOR-LIKE KINASES (CRKs), has two conserved cysteine domains in their extracellular part $(\mathrm{C}-2 \mathrm{x}-\mathrm{C}-8 \mathrm{x}-\mathrm{C}$; DUF26 domain). The structure of the extracellular domain of CRKs, mutant phenotypes, gene expression analysis, and thorough comprehensive transcriptional and phenotypic analysis of CRK mutants has led us and others to imply that CRKs could be involved in apoplastic ROS sensing [37, 38, 47-50].

In addition to modification of extracellular domains of receptors, apoplastic secreted peptides could sense ROS (Figure 1). We have identified the extracellular GRIM REAPER (GRI) protein as a regulator of ROS-induced cell death [26]. A leucine-rich repeat (LRR) receptor-like kinase POLLEN-SPECIFIC RECEPTOR-LIKE KINASE 5 (PRK5) perceives a short peptide proteolytically cleaved from GRI [33]. In Arabidopsis leaves this small GRI-peptide is sufficient to induce cell death that requires ROS. Thus, GRI, and GRIrelated apoplastic proteins could act as a novel class of sensory proteins mediating ROS-dependent information from the apoplast to nucleus and chloroplast.

\section{Apoplast to Nucleus Signaling}

Plants show several different responses downstream of sensing the apoplastic ROS. These range from activation of several signaling cascades and changes in gene expression to induction of programmed cell death. However, most of these responses cannot be regarded as direct or fast responses and involve complex interactions between different signaling systems. These signaling systems include second messengers such as $\mathrm{Ca}^{2+}$ connected to $\mathrm{Ca}^{2+}$-dependent protein kinases, MAP kinase cascades, hormonal signaling, and specific transcription factors that all affect the changes in gene expression detectable after apoplastic ROS sensing [47]. Arabidopsis RCD1 (Figure 2(a)), a gene/protein we originally identified in a mutant screen for ozone sensitivity [23,51], is a member of a nuclear-localized, plant specific small SRO protein family whose members are involved in protein-protein interactions of still unknown mechanistic function [52]. Our recent 
results demonstrate that RCD1 seems to be involved in signaling networks that regulate quantitative changes in gene expression in response to ROS [53].

The $r c d 1$ mutant has defective control of cell death in response to apoplastic ROS. We and others have shown that RCD1 and the other SRO proteins are also involved in several developmental and inducible processes. The protein family is highly conserved in all land plants and is involved, in addition to ROS-related processes, in other abiotic stresses [54-56], in biotic stresses and pathogen defense [57], and in developmental processes such as xylem differentiation [58] and embryo development [52, 58, 59]. We recently demonstrated that all Brassicaceae have two paralogs, RCD1 and SRO1, as a result of recent partial genome duplication, while other land plants have only one orthologous protein [35]. In Arabidopsis, RCD1 and SRO1 have partially redundant function, but in the double mutant, where both paralogs are deficient, embryo development is severely affected resulting in almost unviable seeds that can be barely germinated under artificial conditions-normal germination that does not happen due to malformed embryo [52, 56, 59]. Thus, in Arabidopsis RCD1 and SRO1 can be regarded as essential proteins and presence of at least one of them is necessary for normal development. However, the molecular mechanisms by which RCD1 and SROs control plant acclimation and development have just begun to be revealed. A hypothetical model for the role of RCD1 as a positive and negative coregulator of transcription is shown in Figure 2.

The RCD protein has N-terminal nuclear localization sequences, a WWE domain found in proteins involved in ubiquitination and ADP ribosylation, a "PARP" domain poly(ADP-ribosyl)ation domain, which does not have PARP activity [34]), and a C-terminal protein-protein interaction domain (Figure 2(a)). The WWE domain is a common interaction module in proteins involved in posttranslational regulation of protein stability by ubiquitination and ADP ribosylation and has been shown to bind the iso-ADP-ribose moiety in poly(ADP-ribose) [60]. Poly(ADP-ribosyl)ation is a reversible posttranslational modification that has sometimes been compared to protein phosphorylation; the poly(ADPribose) polymerase (PARP) adds ADP-ribose units from $\mathrm{NAD}^{+}$to a target and the poly(ADP-ribose) glycohydrolase (PARG) removes them (Figure 2(b)). Since the WWE domain present in several animal proteins has been demonstrated to bind PAR [60], there is also a reason to believe that the only plant WWE domain-containing proteins, RCD1 and SRO1, would do the same (Figure 2(b)).

We defined in the C-terminus of RCD1 and other SRO proteins a novel and conserved protein-protein interaction domain, RST (RCD1, SRO, and TAF4) (Figure 2(a)), through which RCD1 and SROs interact with specific transcription factors $[35,52]$. However, the significance of this interaction is unknown. The most prominent RCD1-interacting protein through the RST domain is DREB2A (and also DREB2B and DREB2C, but no other member of the DREB2-protein family), a transcription factor of central importance in plant acclimation to several abiotic stresses in the ABAindependent signaling pathway [61]. The DREB2A B and C proteins have a specific domain which we showed to interact with RCD1 [34]. This domain is completely located in an exon that is a subject to developmental alternative splicing; for example, the domain is absent in DREB2A in leaves approaching senescence. Conserved amino acid residues within the RCD1-interaction motif in DREB2A 2B and 2C are required for the interaction with RCD1 [34]. Thus the interaction between RCD1 and at least some transcription factors is very specific and regulated by both partners.

The RST protein-protein interaction domain [35] is present, in addition to RCD1/SROs, also in plant TAF4protein (TAF = TATA-binding protein-associated factors), a subunit of the general transcription factor complex TFIID. TFIID consists of the TATA-binding protein (TBP) and 1213 TBP-associated factors (TAFs). TFIID forms the heart of the preinitiation complex and DNA-binding proteins directly contact TFIID to stimulate the rate of transcription of target genes. In the same location where plant TAF4 has the RST domain, the animal TAF4 protein contains an ETO domain (originally described in the eight twenty-one protein), which is a docking platform for positive and negative regulators of transcription. In Drosophila, for example, the ETO domain of TAF4 interacts with transcription factors and links them physically with TFIID [62]. However, it is not expected that RCD1 would be part of the TFIID complex in plants. The plant TAF4 protein interacts with TFIID with its C-terminal TAF domain, leaving the centrally located RST domain freely exposed to, for example, interaction with transcription factors. A central question is whether the RST domain in plant TAF4 and also in RCD1 and SROs is similarly a docking domain as ETO is in animals, and whether the RST domain in RCD1 is somehow involved in the control of the action of the transcription factors it interacts with (Figures 2(c) and 2(d)). The common nominator of the RCD1-interacting proteins (for which the information exists) is the regulation of protein stability by posttranslational modifications and proteolytic degradation. The RCD1 and SRO1 proteins themselves also appear to be under similar regulation. Thus, one of the questions requiring further experiments is whether RCD1 might be involved as a coregulator in affecting the stability of the interacting transcription factors and at the same time control their access to the chromatin (Figures 2(c) and 2(d)).

\section{Interplay between Apoplastic and Organellar ROS Signaling Mechanisms}

The two important research fields for plant productivity, photosynthesis, and stress tolerance/acclimation are generally investigated each in isolation. It is however increasingly clear that biotic and abiotic stresses are perceived in both the chloroplast and in apoplast. Moreover, particularly in biotic stress both apoplastic and chloroplastic compartments act in synergy. Indeed, the signaling networks and feedback regulation loops generated by sensing of external changes in the apoplast are likely to be transmitted via chloroplasts further to the nucleus. For example, double mutant analysis suggests a role for RCD1 in retrograde signaling from multiple organelles. We have shown that the $r c d 1$ mutant is sensitive to 


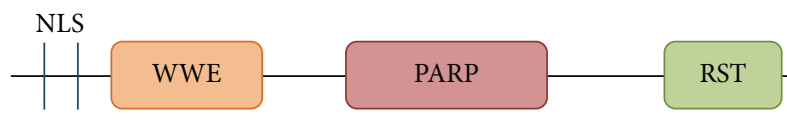

(a)

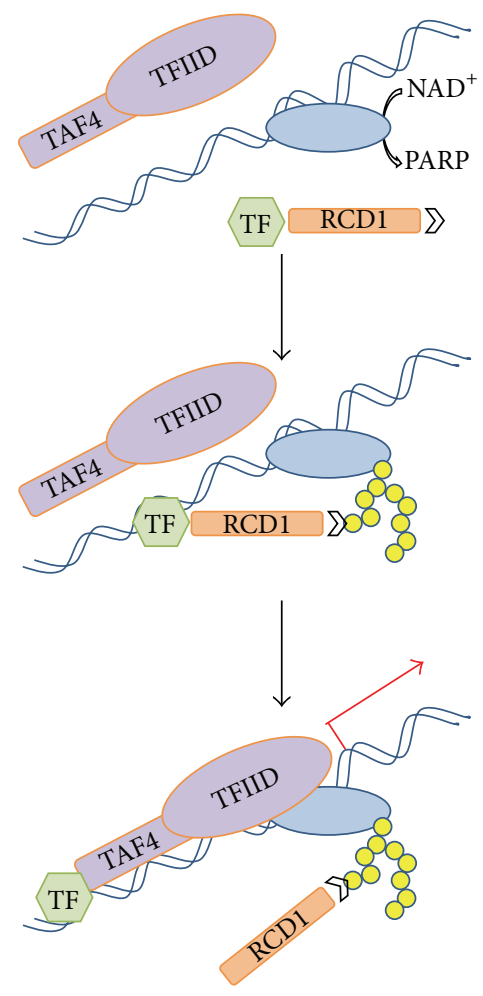

(c)

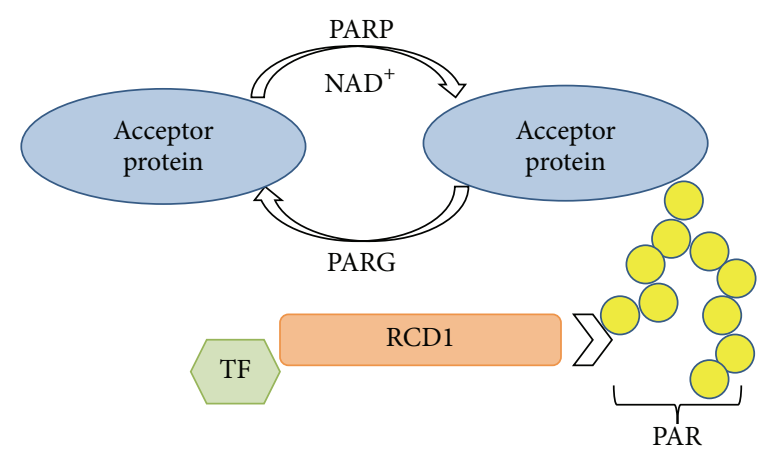

ADP-ribose

(b)
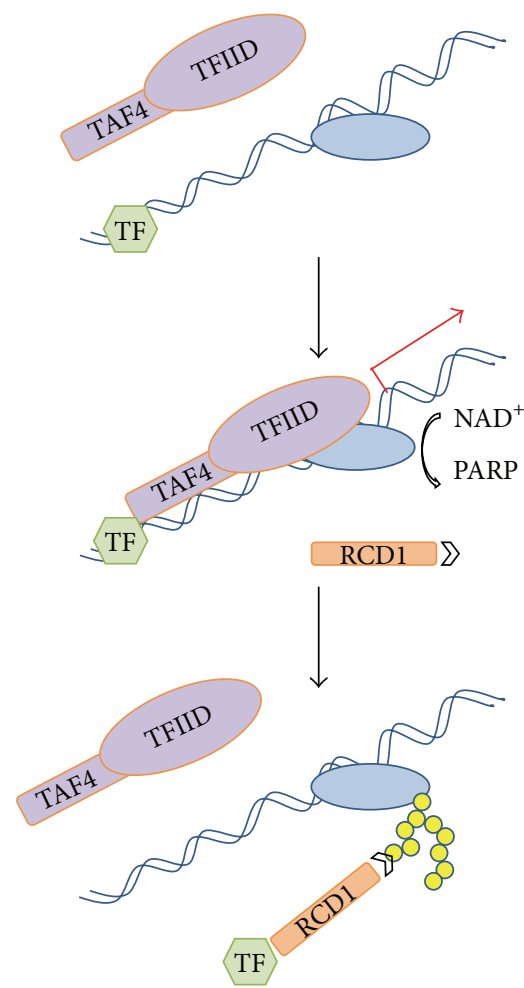

(d)

FIGURE 2: The protein domain structure of RCD1 and a hypothetical model of its action in the regulation of gene expression. (a) The RCD1 protein contains nuclear localization sequences (NLS), a N-terminal WWE-domain, and a PARP (poly(ADP) ribose polymerase) domain, however, without PARP activity [34], and a C-terminal protein-protein interaction domain RST which mediates specific interaction with several transcription factors [35]. (b) Poly(ADP)ribosylation is mediated by the activity of PARP (poly(ADP)ribose polymerase), which transfers ADP-ribose from $\mathrm{NAD}^{+}$to a target protein forming poly(ADP) ribose (PAR). PAR can be removed by PARG (PAR glycohydrolase). RCD1 is assumed to bind PARsylated proteins. (c) Hypothetical model for RCD1 as a positive regulator of specific transcription: RCD1 interacts through the RST-domain with specific transcription factors, binds PARsylated nuclear protein, and transports the interacting transcription factors to a target gene, where they bind to chromatin. The general transcription factor complex TFIID interacts with the specific transcription factor through the RST domain in TAF4, allows the binding of RNA polymerase, and initiates transcription. (d) Hypothetical model for RCD1 as a negative regulator of transcription: the general transcription factor complex TFIID interacts with the specific transcription factor through the RST domain in TAF4 and allows the binding of RNA polymerase to initiate transcription. PARP synthesizes PAR to a chromatin protein allowing RCD1 to interact with chromatin and compete through the RST domain for interaction of a specific transcription factor with TAF4 in the TFIID complex. 
apoplastic ROS but considerably more tolerant to the lightinduced formation of ROS at photosystem I in the chloroplast [23]. Analysis of the photosynthetic functions in $r c d 1$ also suggests that the mutant could be continuously acclimated to high light stress thus linking RCD1 to the regulation of both apoplastic and chloroplastic ROS. ROS formation and signaling in these two subcellular compartments are linked also in many other ways, however, mostly by indirect evidence, and thus this interaction is one of the important topics to address in ROS signaling in the future.

Our results indicate that a short apoplastic ROS burst induces ROS formation in the chloroplasts of guard cells concomitantly with the rapid and transient decrease in stomatal conductance [42]. However, the production mechanism and regulation of this chloroplastic ROS accumulation is unknown and requires further elucidation. It was shown recently that the elicitor-induced stomatal closure and $\mathrm{H}_{2} \mathrm{O}_{2}$ accumulation induced by pathogens were significantly reduced in the cas-1 mutantdeficient in chloroplast thylakoidbound CAS-protein, which is crucial for proper stomatal regulation [63]. CAS was originally described as calcium sensor residing in plasma membrane $[64,65]$ but later shown to be a chloroplast thylakoid protein that modulates cytoplasmic $\mathrm{Ca}^{2+}$ concentration $[66,67]$. Pathogen elicitors (flg22 and chitin) evoked a rapid transient increase in chloroplast stromal $\mathrm{Ca}^{2+}$ concentration and this flg22-induced stromal $\mathrm{Ca}^{2+}$ oscillation was partially dependent on CAS [63]. These data suggest that CAS could be involved in the regulation of chloroplastic ROS production after sensing of and signaling from apoplastic ROS. The electron transport chains of the chloroplasts are a logical source for ROS formation and CAS, together with its interacting components in both linear and cyclic electron transfer pathways, could regulate the processes involved. In these interactions, production of ${ }^{1} \mathrm{O}_{2}$ in the lightharvesting antenna of photosystem II (LHCII) is likely to carry out critical functions, and tightly regulated photoprotective mechanisms are therefore intrinsically connected with the plants' defense programs $[63,67-70]$. Similarly to the current picture in apoplastic ROS signaling, the identity of chloroplast ROS sensors, their functional characteristics, and downstream signaling components remain to be established in future studies.

Our and another mutant screen for ozone sensitivity identified a chloroplast inner envelope membrane protein RCD2/LCD1 [25, 71]. Later $r c d 2$ and $l c d 1$ were shown to be alleles of a classical Arabidopsis mutant reticulata (re) [72]. The RETICULATA protein is apparently a component in apoplast-chloroplast ROS signaling (Figure 1). We have shown that the extent of ozone-induced cell death in re leaves is light intensity dependent, being more severe under moderately high light and less evident under low light intensity [25]. We also found that the re mutant displays reticulated leaf pigmentation with pale green mesophyll cells and dark green bundle sheath cells that, paradoxically, under long day conditions show constitutive accumulation of ROS $[25,73]$. Such pattern of ROS staining is commonly observed in suddenly light stress exposed plants $[74,75]$. Although the underlying molecular mechanisms call for further analysis, these findings suggest that RE controls the outcomes of apoplast-chloroplast ROS signaling interplay.

Perhaps associated with its indisputable role in ROS signaling, RE is also required to maintain amino acid metabolism in chloroplasts and consequently, the ability to synthesize precursors for the plant hormones SA and auxin [73]. Based on this, we can position RE in regulatory network which functionally interconnects photoperiod and hormonal interactions with cross-compartmental ROS signaling which may trigger stress-induced morphogenic responses and activate protective mechanisms in plants [73, 76-78].

The formation of photorespiratory ROS signals in the peroxisomes seems to play a major role in photoperioddependent signaling $[77,78]$. Similarly to re, day length also conditions the ROS-dependent phenotype of Arabidopsis CATALASE2 (CAT2) knock-out mutants which accumulate photorespiratory $\mathrm{H}_{2} \mathrm{O}_{2}$ in the peroxisomes [79]. Knockout cat 2 plants show constitutive SA-dependent defense responses and cell death under long-day conditions. However, under short-day photoperiods SA signaling and cell death are not induced in the cat 2 mutants, despite of the prevalence of oxidative stress [78-80]. We have shown with a combination of genetic, proteomic, and metabolomic analyses that in the cat 2 mutant a cytoplasmic protein phosphatase $2 \mathrm{~A}$ regulatory subunit $\mathrm{B}^{\prime} \gamma\left(\mathrm{PP} 2 \mathrm{~A}-\mathrm{B}^{\prime} \gamma\right)$ is required to suppress the day length-dependent, salicylic acid mediated pathogenesis responses triggered by organellar ROS signals [78, 81]. The molecular targets of PP2A phosphatases are likely to involve both metabolic enzymes and signaling components with well-known roles in cellular ROS homeostasis [78, 81]. PP2A phosphatases are predominantly trimeric and consist of a catalytic subunit $C$, a scaffold subunit $A$, and a variable regulatory subunit $B$, which essentially determines the target specificity of PP2A holoenzymes. Similarly to knockdown of PP2A-B ${ }^{\prime} \gamma$, overexpression of a calcium-dependent protein kinase AtCPK1 triggers constitutive SA signaling responses in Arabidopsis leaves [81, 82]. This raises a question whether PP2A phosphatases could act as the long sought upstream regulatory components that control CPK activity to prevent overexaggeration of stress reactions in plants [83].

\section{Regulation of ROS Signaling through Reversible Protein Phosphorylation}

Although reversible protein phosphorylation is a fundamental mechanism of cellular regulation, comprehensive view to the identities of kinase-phosphatase pairs with counteracting effects on stress responses in plants remains to be established. The anion channel SLAC1, as well as the protein kinase SnRK2.6/OST1, and the PP2C protein phosphatases ABI1 and $\mathrm{ABI} 2$ are required for the regulation of stomatal closure by ROS [42]. After the electrophysiological demonstration of the existence of guard cell anion channels 20 years ago, pharmacological experiments suggested that they are activated by phosphorylation. The identification of the SLAC1 anion channel with the ozone and $\mathrm{CO}_{2}$ sensitive mutants allowed proving this also experimentally. We and others $[42,84,85]$ have shown both with heterologous expression 
of SLAC1 in Xenopus oocytes and in vivo that the protein kinase SnRK2.6/OST1 can phosphorylate several amino acid residues at the soluble $\mathrm{N}$-terminus of SLACl. We identified with the use of "Tilling" technique in the soluble N-terminus of SLAC1 mutations in conserved Ser/Thr residues that have a strong prediction to be targets for phosphorylation-stomatal function was impaired in these mutants [42]. Experiments with Xenopus oocytes have shown that, when coexpressed with SLAC1, in addition to SnRK2.6/OST1 $[84,85]$, also $\mathrm{Ca}^{2+}$-dependent protein kinases activate SLACl-dependent ion currents in oocytes by phosphorylation of more different amino acid residues than SnRK2.6/OST1 [86, 87]. This demonstrates that several protein kinases can activate SLAC1dependent anion currents with specific phosphorylation, which agrees well with the indirect evidence for several independent regulatory mechanisms for stomatal movements and has paved the way forward for further studies in stomatal regulation-including the elucidation of the mechanistic role of ROS therein.

\section{Outlook}

All living organisms react to both internal and external cues and reprogram their physiology and gene expression based on the information received; this is the central feature of growth and development as well as of environmental adaptation. These processes use the same basic scheme starting from the perception and recognition of a signal followed by the transmission of the signal leading to specific changes in physiology, gene expression, metabolite content, and growth. ROS have been established as signaling molecules in several aspects of plant life. However, the regulatory mechanisms at the biochemical level, the mechanisms downstream of ROS perception, and especially the communication and interaction between different subcellular compartments in ROS signaling are still poorly understood. Due to the importance of ROS as signaling molecules, it is central to modern plant biology to obtain a comprehensive understanding of the processes where ROS have regulatory roles. This is not only of great interest from physiological, molecular, and evolutionary research perspectives but is also of great importance for practical applications. The ultimate goal in identifying and elucidating the components of ROS signaling with ozone as a tool is to define the mechanisms and protein complexes that connect extracellular ROS-specific signals to chromatin restructuring and transcriptional regulation (extracellular space-plasma membrane-cytoplasm/chloroplast-nucleus continuum). This aims at building comprehensive plant protein interaction pathways and networks in ROS signaling and responses requires a combination of transcriptomics and proteomics approaches with analysis of protein-protein interactions and bioinformatics/systems biology, and mutant analysis.

\section{Conflict of Interests}

The authors declare that there is no conflict of interests regarding the publication of this paper.

\section{References}

[1] R. Mittler, S. Vanderauwera, M. Gollery, and F. van Breusegem, "Reactive oxygen gene network of plants," Trends in Plant Science, vol. 9, no. 10, pp. 490-498, 2004.

[2] C. H. Foyer and G. Noctor, "Redox regulation in photosynthetic organisms: signaling, acclimation, and practical implications," Antioxidants \& Redox Signaling, vol. 11, no. 4, pp. 861-905, 2009.

[3] N. Suzuki, S. Koussevitzky, R. Mittler, and G. Miller, "ROS and redox signalling in the response of plants to abiotic stress," Plant, Cell and Environment, vol. 35, no. 2, pp. 259-270, 2012.

[4] R. Mittler, S. Vanderauwera, N. Suzuki et al., "ROS signaling: the new wave?” Trends in Plant Science, vol. 16, no. 6, pp. 300-309, 2011.

[5] M. Sierla, M. Rahikainen, J. Salojärvi, J. Kangasjärvi, and S. Kangasjärvi, "Apoplastic and chloroplastic redox signaling networks in plant stress responses," Antioxidants \& Redox Signaling, vol. 18, no. 16, pp. 2220-2239, 2013.

[6] J. Kangasjarvi, J. Talvinen, M. Utriainen, and R. Karjalainen, "Plant defence systems induced by ozone," Plant, Cell and Environment, vol. 17, no. 7, pp. 783-794, 1994.

[7] G. Miller, V. Shulaev, and R. Mittler, "Reactive oxygen signaling and abiotic stress," Physiologia Plantarum, vol. 133, no. 3, pp. 481-489, 2008

[8] L. Vaahtera, M. Brosché, M. Wrzaczek, and J. Kangasjärvi, "Specificity in ROS signaling and transcript signatures," Antioxidants \& Redox Signaling, 2014.

[9] A. Shapiguzov, J. P. Vainonen, M. Wrzaczek, and J. Kangasjärvi, "ROS-talk-how the apoplast, the chloroplast, and the nucleus get the message through," Frontiers in Plant Science, vol. 3, article 292, 2012.

[10] S. Karpiński, M. Szechyńska-Hebda, W. Wituszyńska, and P. Burdiak, "Light acclimation, retrograde signalling, cell death and immune defences in plants," Plant, Cell and Environment, vol. 36, no. 4, pp. 736-744, 2013.

[11] M. J. Considine and C. Foyer, "Redox regulation of plant development," Antioxidants \& Redox Signaling, 2014.

[12] J. Dowdle, T. Ishikawa, S. Gatzek, S. Rolinski, and N. Smirnoff, "Two genes in Arabidopsis thaliana encoding GDP-L-galactose phosphorylase are required for ascorbate biosynthesis and seedling viability," Plant Journal, vol. 52, no. 4, pp. 673-689, 2007.

[13] S. Kangasjärvi, A. Lepistö, K. Hännikäinen et al., "Diverse roles for chloroplast stromal and thylakoid-bound ascorbate peroxidases in plant stress responses," Biochemical Journal, vol. 412, no. 2, pp. 275-285, 2008.

[14] H. Fahnenstich, T. E. Scarpeci, E. M. Valle, U.-I. Flügge, and V. G. Maurino, "Generation of hydrogen peroxide in chloroplasts of arabidopsis overexpressing glycolate oxidase as an inducible system to study oxidative stress," Plant Physiology, vol. 148, no. 2, pp. 719-729, 2008.

[15] A. Mhamdi, G. Queval, S. Chaouch, S. Vanderauwera, F. van Breusegem, and G. Noctor, "Catalase function in plants: a focus on Arabidopsis mutants as stress-mimic models," Journal of Experimental Botany, vol. 61, no. 15, pp. 4197-4220, 2010.

[16] J. Kangasjärvi, P. Jaspers, and H. Kollist, "Signalling and cell death in ozone-exposed plants," Plant, Cell and Environment, vol. 28 , no. 8, pp. 1021-1036, 2005.

[17] J. P. Vainonen and J. Kangasjärvi, "Plant signaling in acute ozone exposure," Plant Cell and Environment, 2014. 
[18] S. V. Krupa, "Joint effects of elevated levels of ultraviolet-B radiation, carbon dioxide and ozone on plants," Photochemistry and Photobiology, vol. 78, no. 6, pp. 535-542, 2003.

[19] E. A. Ainsworth, C. R. Yendrek, S. Sitch, W. J. Collins, and L. D. Emberson, "The effects of tropospheric ozone on net primary productivity and implications for climate change," Annual Review of Plant Biology, vol. 63, pp. 637-661, 2012.

[20] M. V. Rao, J. R. Koch, and K. R. Davis, “Ozone: a tool for probing programmed cell death in plants," Plant Molecular Biology, vol. 44, no. 3, pp. 345-358, 2000.

[21] S. Pasqualini, C. Piccioni, L. Reale, L. Ederli, G. D. Della Torre, and F. Ferranti, "Ozone-induced cell death in tobacco cultivar Bel W3 plants. The role of programmed cell death in lesion formation," Plant Physiology, vol. 133, no. 3, pp. 1122-1134, 2003.

[22] K. Overmyer, M. Brosché, and J. Kangasjärvi, "Reactive oxygen species and hormonal control of cell death," Trends in Plant Science, vol. 8, no. 7, pp. 335-342, 2003.

[23] R. Ahlfors, S. Lång, K. Overmyer et al., "Arabidopsis RADICALINDUCED CELL DEATH1 belongs to the WWE proteinprotein interaction domain protein family and modulates abscisic acid, ethylene, and methyl jasmonate responses," The Plant Cell, vol. 16, no. 7, pp. 1925-1937, 2004.

[24] T. Vahisalu, H. Kollist, Y.-F. Wang et al., "SLAC1 is required for plant guard cell S-type anion channel function in stomatal signalling," Nature, vol. 452, no. 7186, pp. 487-491, 2008.

[25] K. Overmyer, H. Kollist, H. Tuominen et al., "Complex phenotypic profiles leading to ozone sensitivity in Arabidopsis thaliana mutants," Plant, Cell and Environment, vol. 31, no. 9, pp. 12371249, 2008.

[26] M. Wrzaczek, M. Brosché, H. Kollist, and J. Kangasjärvi, "Arabidopsis GRI is involved in the regulation of cell death induced by extracellular ROS," Proceedings of the National Academy of Sciences of the United States of America, vol. 106, no. 13, pp. 5412-5417, 2009.

[27] P. L. Conklin, S. A. Saracco, S. R. Norris, and R. L. Last, "Identification of ascorbic acid-deficient Arabidopsis thaliana mutants," Genetics, vol. 154, no. 2, pp. 847-856, 2000.

[28] P. L. Conklin, E. H. Williams, and R. L. Last, "Environmental stress sensitivity of an ascorbic acid-deficient arabidopsis mutant," Proceedings of the National Academy of Sciences of the United States of America, vol. 93, no. 18, pp. 9970-9974, 1996.

[29] J. Negi, O. Matsuda, T. Nagasawa et al., "CO2 regulator SLAC1 and its homologues are essential for anion homeostasis in plant cells," Nature, vol. 452, no. 7186, pp. 483-486, 2008.

[30] S. Saji, S. Bathula, A. Kubo et al., "Disruption of a gene encoding C4-dicarboxylate transporter-like protein increases ozone sensitivity through deregulation of the stomatal response in Arabidopsis thaliana," Plant and Cell Physiology, vol. 49, no. 1, pp. 2-10, 2008.

[31] M. Brosché, E. Merilo, F. Mayer et al., "Natural variation in ozone sensitivity among Arabidopsis thaliana accessions and its relation to stomatal conductance," Plant, Cell and Environment, vol. 33, no. 6, pp. 914-925, 2010.

[32] G. L. Wheeler and C. Brownlee, " $\mathrm{Ca}^{2+}$ signalling in plants and green algae-changing channels," Trends in Plant Science, vol. 13, no. 9, pp. 506-514, 2008.

[33] M. Wrzaczek, J. P. Vainonen, H. Help et al., "Arabidopsis GRIM REAPER functions through metacaspase processing and receptor-ligand interaction," submitted. In press.
[34] J. P. Vainonen, P. Jaspers, M. Wrzaczek et al., "RCD1-DREB2A interaction in leaf senescence and stress responses in Arabidopsis thaliana," Biochemical Journal, vol. 442, no. 3, pp. 573-581, 2012.

[35] P. Jaspers, K. Overmyer, M. Wrzaczek et al., "The RST and PARP-like domain containing SRO protein family: analysis of protein structure, function and conservation in land plants," BMC Genomics, vol. 11, article 170, 2010.

[36] G. Miller, K. Schlauch, R. Tam et al., "The plant NADPH oxidase RBOHD mediates rapid systemic signaling in response to diverse stimuli," Science Signaling, vol. 2, no. 84, Article ID ra45, 2009.

[37] M. Wrzaczek, M. Brosché, J. Salojärvi et al., "Transcriptional regulation of the CRK/DUF26 group of receptor-like protein kinases by ozone and plant hormones in Arabidopsis," BMC Plant Biology, vol. 10, article 95, 2010.

[38] N. Idänheimo, A. Gauthier, J. Salojärvi et al., “The Arabidopsis thaliana cysteine-rich receptor-like Kinases CRK6 and CRK7 protect against apoplastic oxidative stress," Biochemical and Biophysical Research Communications, vol. 445, pp. 457-462, 2014.

[39] C. Garcia-Mata, J. Wang, P. Gajdanowicz et al., "A minimal cysteine motif required to activate the SKOR $\mathrm{K}^{+}$channel of arabidopsis by the reactive oxygen species $\mathrm{H}_{2} \mathrm{O}_{2}$," The Journal of Biological Chemistry, vol. 285, no. 38, pp. 29286-29294, 2010.

[40] H. Clayton, M. R. Knight, H. Knight, M. R. McAinsh, and A. M. Hetherington, "Dissection of the ozone-induced calcium signature," Plant Journal, vol. 17, no. 5, pp. 575-579, 1999.

[41] N. H. Evans, M. R. McAinsh, A. M. Hetherington, and M. R. Knight, "ROS perception in Arabidopsis thaliana: the ozoneinduced calcium response," Plant Journal, vol. 41, no. 4, pp. 615626, 2005.

[42] T. Vahisalu, I. Puzõrjova, M. Brosché et al., "Ozone-triggered rapid stomatal response involves the production of reactive oxygen species, and is controlled by SLAC1 and OST1," Plant Journal, vol. 62, no. 3, pp. 442-453, 2010.

[43] M. Melotto, W. Underwood, J. Koczan, K. Nomura, and S. Y. He, "Plant stomata function in innate immunity against bacterial invasion," Cell, vol. 126, no. 5, pp. 969-980, 2006.

[44] S.-H. Shiu and A. B. Bleecker, "Expansion of the receptorlike kinase/Pelle gene family and receptor-like proteins in Arabidopsis," Plant Physiology, vol. 132, no. 2, pp. 530-543, 2003.

[45] S.-H. Shiu, W. M. Karlowski, R. Pan, Y.-H. Tzeng, K. F. X. Mayer, and W.-H. Li, "Comparative analysis of the receptor-like kinase family in Arabidopsis and rice," The Plant Cell, vol. 16, no. 5, pp. 1220-1234, 2004.

[46] M. D. Lehti-Shiu, C. Zou, K. Hanada, and S.-H. Shiu, "Evolutionary history and stress regulation of plant receptor-like kinase/pelle genes," Plant Physiology, vol. 150, no. 1, pp. 12-26, 2009.

[47] M. Wrzaczek, M. Brosché, and J. Kangasjärvi, "ROS signaling loops-production, perception, regulation," Current Opinion in Plant Biology, vol. 16, no. 5, pp. 575-582, 2013.

[48] P. Czernic, B. Visser, W. Sun et al., "Characterization of an versus Arabidopsis thaliana receptor-like protein kinase gene activated by oxidative stress and pathogen attack," Plant Journal, vol. 18, no. 3, pp. 321-327, 1999.

[49] K. Chen, B. Fan, L. Du, and Z. Chen, "Activation of hypersensitive cell death by pathogen-induced receptor-like protein kinases from Arabidopsis," Plant Molecular Biology, vol. 56, no. 2, pp. 271-283, 2004. 
[50] S. Munné-Bosch, G. Queval, and C. H. Foyer, “The impact of global change factors on redox signaling underpinning stress tolerance," Plant Physiology, vol. 161, no. 1, pp. 5-19, 2013.

[51] K. Overmyer, H. Tuominen, R. Kettunen et al., "Ozone-sensitive Arabidopsis $r c d 1$ mutant reveals opposite roles for ethylene and jasmonate signaling pathways in regulating superoxidedependent cell death," The Plant Cell, vol. 12, no. 10, pp. 18491862, 2000.

[52] P. Jaspers, T. Blomster, M. Brosché et al., "Unequally redundant RCD1 and SRO1 mediate stress and developmental responses and interact with transcription factors," Plant Journal, vol. 60, no. 2, pp. 268-279, 2009.

[53] M. Brosché, T. Blomster, J. Salojärvi et al., "Transcriptomics and functional genomics of ROS-induced cell death regulation by RADICAL-INDUCED CELL DEATH1," PLoS Genetics, vol. 10, Article ID e1004112, 2014.

[54] O. Borsani, J. Zhu, P. E. Verslues, R. Sunkar, and J.-K. Zhu, "Endogenous siRNAs derived from a pair of natural cisantisense transcripts regulate salt tolerance in Arabidopsis," Cell, vol. 123, no. 7, pp. 1279-1291, 2005.

[55] S. Katiyar-Agrwal, J. Zhu, K. Kim et al., "A pathogen-inducible endogenous siRNA in plant immunity," Proceedings of the National Academy of Sciences USA, vol. 103, no. 49, pp. 1881618821, 2006.

[56] S. Liu, S. Liu, M. Wang et al., "A Wheat SIMILAR TO RCD-ONE gene enhances seedling growth and abiotic stress resistance by modulating redox homeostasis and maintaining genomic integrity," The Plant Cell, vol. 26, no. 1, pp. 164-180, 2014.

[57] Y. Zhu, B. Du, J. Qian, B. Zou, and J. Hua, "Disease resistance gene-induced growth inhibition is enhanced by $r c d 1$ independent of defense activation in Arabidopsis," Plant Physiology, vol. 161, no. 4, pp. 2005-2013, 2013.

[58] E. Pesquet, B. Zhang, A. Gorzsás et al., "Non-cell-autonomous postmortem lignification of tracheary elements in Zinnia elegans," The Plant Cell, vol. 25, no. 4, pp. 1314-1328, 2013.

[59] S. Teotia and R. S. Lamb, "The paralogous genes RADICALINDUCED CELL DEATH1 and SIMILAR TO RCD ONE1 have partially redundant functions during arabidopsis development," Plant Physiology, vol. 151, no. 1, pp. 180-198, 2009.

[60] Z. Wang, G. A. Michaud, Z. Cheng et al., "Recognition of the iso-ADP-ribose moiety in poly(ADP-ribose) by WWE domains suggests a general mechanism for poly (ADP-ribosyl)ationdependent ubiquitination," Genes \& Development, vol. 26, no. 3, pp. 235-240, 2012.

[61] Y. Sakuma, K. Maruyama, Y. Osakabe et al., "Functional analysis of an Arabidopsis transcription factor, DREB2A, involved in drought-responsive gene expression," The Plant Cell, vol. 18, no. 5, pp. 1292-1309, 2006.

[62] K. J. Wright and R. Tjian, "Wnt signaling targets ETO coactivation domain of TAF4/TFIID in vivo," Proceedings of the National Academy of Sciences of the United States of America, vol. 106, no. 1, pp. 55-60, 2009.

[63] H. Nomura, T. Komori, S. Uemura et al., "Chloroplast-mediated activation of plant immune signalling in Arabidopsis," Nature Communications, vol. 3, article 926, 2012.

[64] S. Han, R. Tang, L. K. Anderson, T. E. Woerner, and Z.-M. Pei, "A cell surface receptor mediates extracellular $\mathrm{Ca}^{2+}$ sensing in guard cells," Nature, vol. 425, no. 6954, pp. 196-200, 2003.

[65] R.-H. Tang, S. Han, H. Zheng et al., "Coupling diurnal cytosolic $\mathrm{Ca}^{2+}$ oscillations to the CAS-IP 3 pathway in Arabidopsis," Science, vol. 315, no. 5817, pp. 1423-1426, 2007.
[66] J. P. Vainonen, Y. Sakuragi, S. Stael et al., "Light regulation of $\mathrm{CaS}$, a novel phosphoprotein in the thylakoid membrane of Arabidopsis thaliana," The FEBS Journal, vol. 275, no. 8, pp. 1767$1777,2008$.

[67] S. Weinl, K. Held, K. Schlücking et al., "A plastid protein crucial for $\mathrm{Ca}^{2+}$-regulated stomatal responses," New Phytologist, vol. 179, no. 3, pp. 675-686, 2008.

[68] C. Kim, R. Meskauskiene, K. Apel, and C. Laloi, "No single way to understand singlet oxygen signalling in plants," The EMBO Reports, vol. 9, no. 5, pp. 435-439, 2008.

[69] V. Göhre, A. M. E. Jones, J. Sklenář, S. Robatzek, and A. P. M. Weber, "Molecular crosstalk between PAMP-triggered immunity and photosynthesis," Molecular Plant-Microbe Interactions, vol. 25, no. 8, pp. 1083-1092, 2012.

[70] M. Tikkanen, N. R. Mekala, and E. M. Aro, "Photosystem II photoinhibition-repair cycle protects Photosystem I from irreversible damage," Biochimia et Biophysica Acta, vol. 1837, no. 1, pp. 210-215, 2014.

[71] C. Barth and P. L. Conklin, "The lower cell density of leaf parenchyma in the Arabidopsis thaliana mutant $l c d 1-1$ is associated with increased sensitivity to ozone and virulent Pseudomonas syringae," The Plant Journal, vol. 35, no. 2, pp. 206-218, 2003.

[72] R. González-Bayón, E. A. Kinsman, V. Quesada et al., "Mutations in the RETICULATA gene dramatically alter internal architecture but have little effect on overall organ shape in Arabidopsis leaves," Journal of Experimental Botany, vol. 57, no. 12, pp. 3019-3031, 2006.

[73] J. M. Pérez-Pérez, D. Esteve-Bruna, R. González-Bayón et al., "Functional redundancy and divergence within the Arabidopsis RETICULATA-RELATED gene family," Plant Physiology, vol. 162, no. 2, pp. 589-603, 2013.

[74] S. Karpinski, H. Reynolds, B. Karpinska, G. Wingsle, G. Creissen, and P. Mullineaux, "Systemic signaling and acclimation in response to excess excitation energy in Arabidopsis," Science, vol. 284, no. 5414, pp. 654-657, 1999.

[75] M. Suorsa, S. Järvi, M. Grieco et al., "PROTON GRADIENT REGULATION5 is essential for proper acclimation of Arabidopsis photosystem I to naturally and artificially fluctuating light conditions," The Plant Cell, vol. 24, no. 7, pp. 2934-2948, 2012.

[76] T. Blomster, J. Salojärvi, N. Sipari et al., "Apoplastic reactive oxygen species transiently decrease auxin signaling and cause stress-induced morphogenic response in Arabidopsis," Plant Physiology, vol. 157, no. 4, pp. 1866-1883, 2011.

[77] S. Kangasjärvi, J. Neukermans, S. Li, E.-M. Aro, and G. Noctor, "Photosynthesis, photorespiration, and light signalling in defence responses," Journal of Experimental Botany, vol. 63, no. 4, pp. 1619-1636, 2012.

[78] S. Li, A. Mhamdi, A. Trotta et al., "The protein phosphatase subunit $\mathrm{PP} 2 \mathrm{~A}-\mathrm{B}^{\prime} \gamma$ is required to suppress day length-dependent pathogenesis responses triggered by intracellular oxidative stress," New Phytologist, vol. 202, no. 1, pp. 145-160, 2014.

[79] G. Queval, E. Issakidis-Bourguet, F. A. Hoeberichts et al., "Conditional oxidative stress responses in the Arabidopsis photorespiratory mutant cat 2 demonstrate that redox state is a key modulator of daylength-dependent gene expression, and define photoperiod as a crucial factor in the regulation of $\mathrm{H}_{2} \mathrm{O}_{2}$ induced cell death," Plant Journal, vol. 52, no. 4, pp. 640-657, 2007.

[80] S. Chaouch, G. Queval, S. Vanderauwera et al., "Peroxisomal hydrogen peroxide is coupled to biotic defense responses by 
ISOCHORISMATE SYNTHASE1 in a daylength-related manner," Plant Physiology, vol. 153, no. 4, pp. 1692-1705, 2010.

[81] A. Trotta, M. Wrzaczek, J. Scharte et al., "Regulatory subunit $\mathrm{B}^{\prime} \gamma$ of protein phosphatase $2 \mathrm{~A}$ prevents unnecessary defense reactions under low light in arabidopsis," Plant Physiology, vol. 156, no. 3, pp. 1464-1480, 2011.

[82] M. Coca and B. San Segundo, "AtCPK1 calcium-dependent protein kinase mediates pathogen resistance in Arabidopsis," Plant Journal, vol. 63, no. 3, pp. 526-540, 2010.

[83] P. Schulz, M. Herde, and T. Romeis, "Calcium-dependent protein kinases: hubs in plant stress signaling and development," Plant Physiology, vol. 163, no. 2, pp. 523-530, 2013.

[84] D. Geiger, S. Scherzer, P. Mumm et al., "Activity of guard cell anion channel SLAC1 is controlled by drought-stress signaling kinase-phosphatase pair," Proceedings of the National Academy of Sciences of the United States of America, vol. 106, no. 50, pp. 21425-21430, 2009.

[85] S. C. Lee, W. Lan, B. B. Buchanan, and S. Luan, "A protein kinase-phosphatase pair interacts with an ion channel to regulate ABA signaling in plant guard cells," Proceedings of the National Academy of Sciences of the United States of America, vol. 106, no. 50, pp. 21419-21424, 2009.

[86] D. Geiger, S. Scherzer, P. Mumm et al., "Guard cell anion channel SLAC1 is regulated by CDPK protein kinases with distinct $\mathrm{Ca}^{2+}$ affinities," Proceedings of the National Academy of Sciences of the United States of America, vol. 107, no. 17, pp. 8023-8028, 2010.

[87] B. Brandt, D. E. Brodsky, S. Xue et al., "Reconstitution of abscisic acid activation of SLAC1 anion channel by CPK6 and OST1 kinases and branched ABI1 PP2C phosphatase action," Proceedings of the National Academy of Sciences of the United States of America, vol. 109, no. 26, pp. 10593-10598, 2012. 

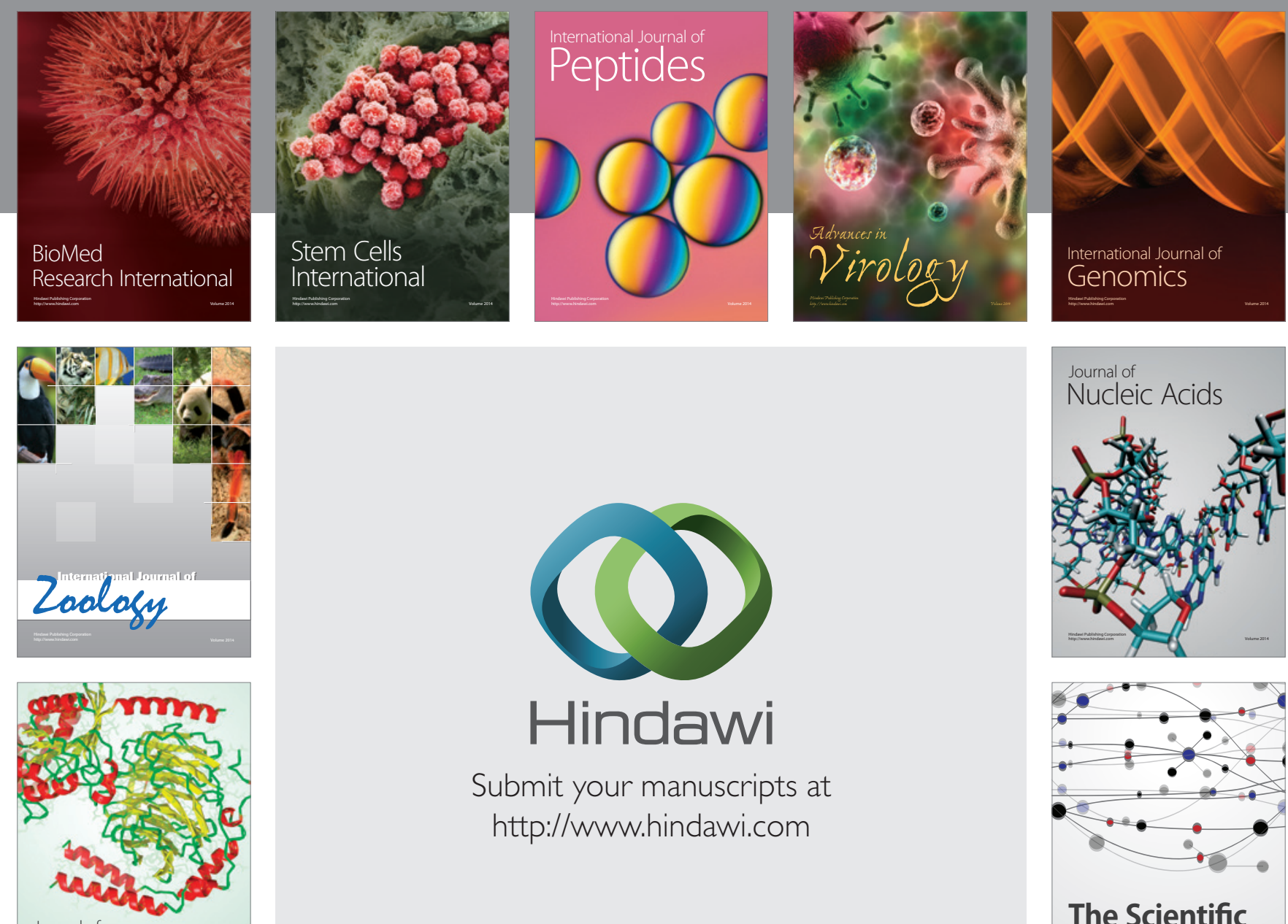

Submit your manuscripts at

http://www.hindawi.com

Journal of
Signal Transduction
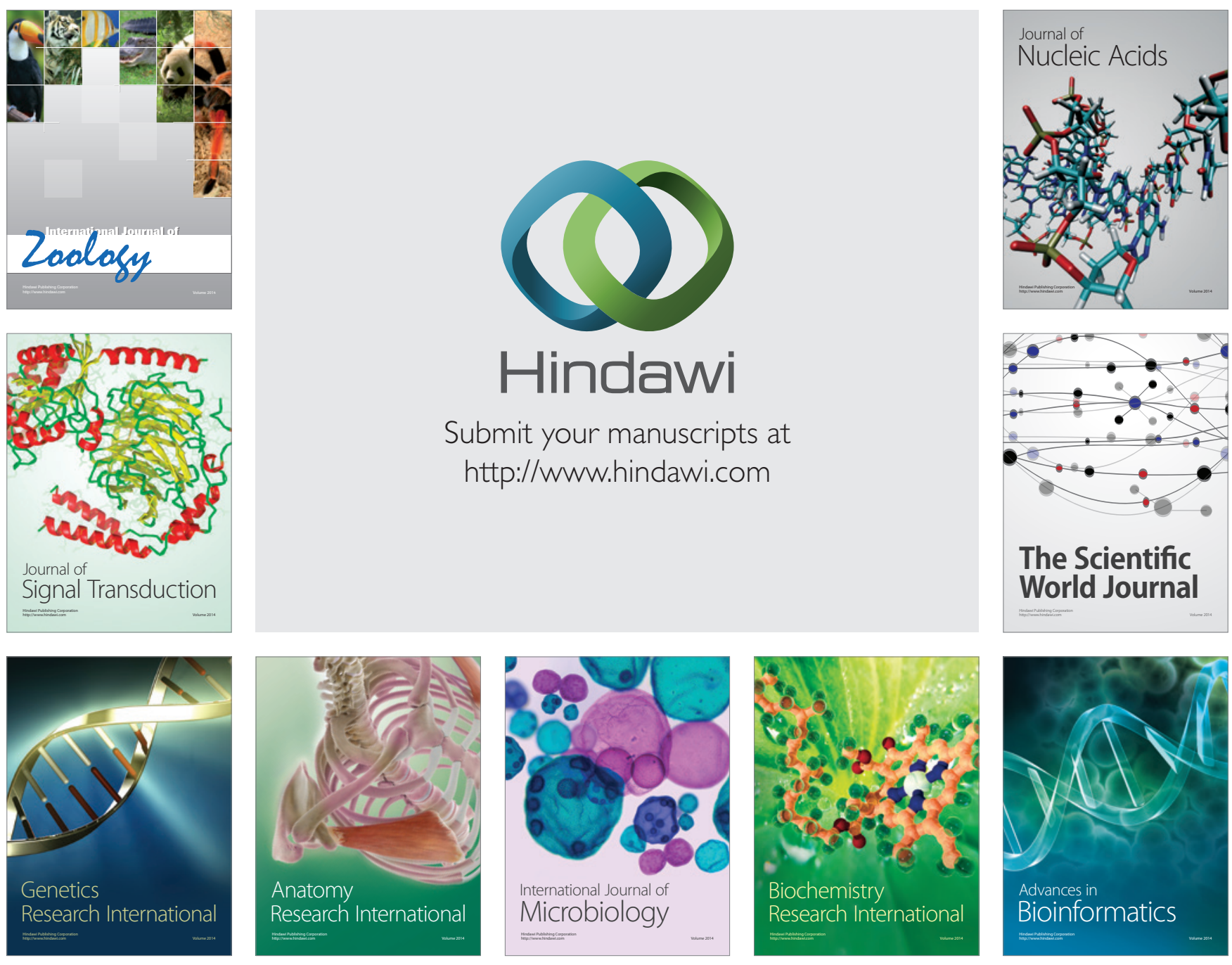

The Scientific World Journal
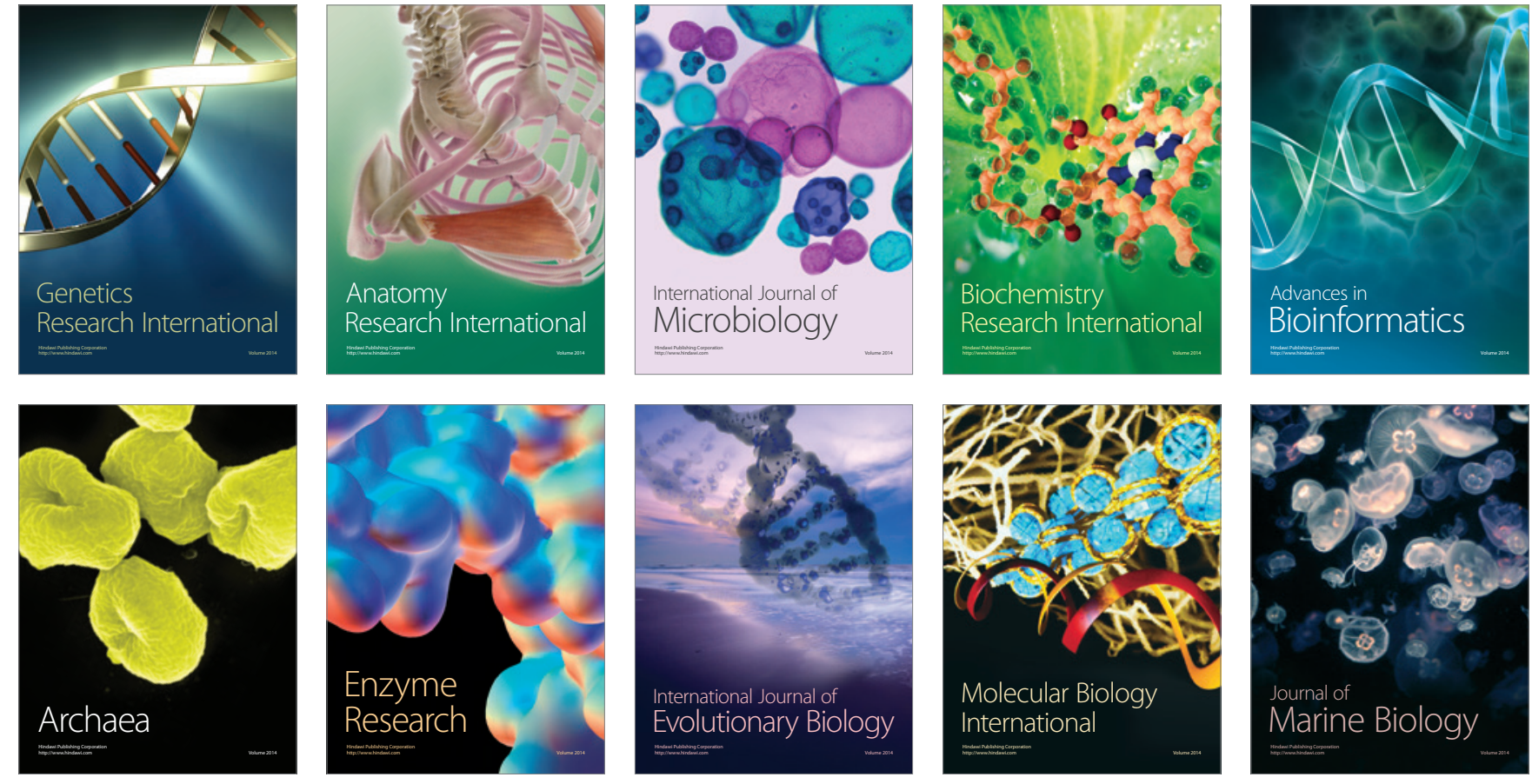